\title{
Morfología del polen de especies neotropicales de Podostemum (Podostemaceae)
}

\author{
Lilian M. Passarelli ${ }^{1}$, Nuncia M. Tur ${ }^{1} \&$ Susana B. Girarde ${ }^{2}$
}

1. Laboratorio de Estudios de Anatomía Vegetal Evolutiva y Sistemática (LEAVES), Facultad de Ciencias Naturales y Museo de La Plata, 64 entre 120 y diagonal 113, B1904 DZB, La Plata, Argentina; 1mpassarelli@yahoo.com.ar; susanagirarde@yahoo.com.ar

2. División Científica Plantas Vasculares, Facultad de Ciencias Naturales y Museo de la Plata, Paseo del Bosque s/n, La Plata, Argentina; tur@fcnym.unlp.edu.ar

\section{Recibido 27-II-2009. Corregido 10-VI-2009. Aceptado 14-VII-2009.}

\begin{abstract}
Pollen morphology of neotropical species of Podostemum (Malpighiales: Podostemaceae). Pollen morphology of neotropical species of Podostemum is described for the first time with light (LM) and scanning electronic microscopes (SEM), using pollen dehydration by critical point. Herbarium specimens under study are P. comatum, P. distichum, P. muelleri and P. rutifolium. Pollen grains are dispersed in dyads. Observed with LM, dyads range from 21 to $31 \mu \mathrm{m}$ in lengh and 12 to $18 \mu \mathrm{m}$ wide. Individual pollen grains are radially symmetrical spheroidal to subprolate, and have a tricolpate aperture. The exine is tectate, psilate, with an infratectal structure formed by simple columella under the tectum. With SEM, an abundant pollen surface coat is observed all over the pollen grains, mainly in the two grains dyad contact zone. This pollen coat would protect the grains from dehydration because the environments in which these plants grow have important water variation and pollination is not zoophilic. The shared dyad wall shows bridges that partially fusion the exines, forming calymate dyads. Some dyads bear the apertures aligned between grains and some not. The transverse condition of the aperture or aperture in " $\mathrm{L}$ " that occurs in the four studied species is described for the first time. It is interpreted as a trend of the genus to espiroaperture. This change in the aperture would be associated with phenology because it is a genus with very short flowering and anthesis, generally a day of anthesis in the dry season. The spiroaperture increases the chances of germination sites and would also have a harmomegata role in an environment with water changes favoring the reproductive success. Walls have a microechinate sculpture, with or without pads at the base of the microechinae. Size of ornamental processes differs, and the colpus membrane has similar ornamentation to that of the non apertural wall, but with larger processes. Individual morphology of pollen grains is similar to that described for other family genera as Apinagia and Mourera. During previous stages of anthesis, the presence of cross tetrads was observed, also a new contribution for this genus. Rev. Biol. Trop. 58 (1): 81-88. Epub 2010 March 01.
\end{abstract}

Key words: pollen, Podostemum - Podostemaceae, dyads, espiroaperture, aquatic plants, neotropical flora.

Las Podostemaceae Rich. ex C.A. Agardh, con unos 46 géneros y aproximadamente unas 270 especies, se considera la familia más grande de angiospermas acuáticas (Philbrick \& Novelo 1995, Cook 1996, Les et al. 1997). Está representada por plantas herbáceas acuáticas, generalmente sumergidas. Son cosmopolitas, de trópicos y subtrópicos. La mayoría de las especies viven en ríos y caidas de agua, adheridas a rocas o troncos rugosos. Muchas especies son endémicas en áreas geográficas pequeñas y de determinados ríos o cataratas.

Estudios recientes basados en análisis moleculares indican que se incluyen en Malpighiales y están relacionadas con Clusiaceae (Hypericaceae). Tristichoideae y Weddellinoideae se aceptan como subfamilias y se consideran distintas de la amplia subfamilia Podostemoideae (Kita \& Kato 2001), en la que se incluye el género Podostemum. Esos autores 
sugieren que la familia se habría originado en América, se habría dispersado luego hacia el Viejo Mundo y que los pertenecientes a Podostemoideae corresponden a dos clados americanos, uno asiático-australiano y uno mascarense. Está bien representada en el neotrópico, con unos 19 géneros y unas 165 especies. Poseen flores bisexuales, solitarias o reunidas en inflorescencias incluidas en una espata con hasta 20 flores. Estas son pequeñas, actinomorfas o zigomorfas, bracteoladas, hermafroditas, con 2-5 tépalos libres a más o menos connatos, 2-3 estilos, 1-4 estambres con los filamentos libres o connatos y anteras 2-4 esporangiadas con endotecio fibroso, tapete glandular y dehiscencia introrsa. El ovario es súpero, con 2-3 lóculos y el fruto es una cápsula septicida que produce numerosas semillas sin endosperma. El polen se dispersa en forma de granos solitarios o díades.

El género Podostemum Michx comprende cerca de 11 especies neotropicales, cuatro de las cuales, Podostemum comatum Hicken, $P$. distichum Chodat et Vischer, P. muelleri Warm y $P$. rutifolium Warm crecen en las selvas tropicales y subtropicales del NE de Argentina (Philbrick \& Novelo 2004).

Ancíbor (1990) estudió la anatomía de varias especies argentinas del género Podostemum. Sin embargo el conocimiento del género es insuficiente y en aspectos palinológicos sólo se conocen los de O'Neill et al.(1997) sobre P. ceratophyllum Michx., en tanto que nada se sabe sobre la palinología de las otras especies.

En este trabajo se ha estudiado el polen de cuatro especies, Podostemum comatum, $P$. distichum, P. muelleri (incluyendo $P$. uruguayense Warm.) y P. rutifolium con el objetivo el contribuir a su mejor conocimiento y relacionar los resultados de los aspectos palinológicos con la morfología de las especies del género.

\section{MATERIALES Y MÉTODOS}

Se trabajó con material de herbario. Los herbarios consultados fueron - Swedish Museum of Natural History (C), Stockholm; Herbario del Instituto de Botánica del
Nordeste, Corrientes (CTES); Herbario del Instituto Fundación Miguel Lillo (LIL), San Miguel de Tucumán; Herbario del Museo Argentino de Ciencias Naturales Bernardino Rivadavia (MCNS) (BA); Herbario Gaspar Xuarez, Facultad de Agronomía de la Universidad de Buenos Aires (BAA); Herbario de la Facultad de Ciencias Naturales y Museo de La Plata (LP); The New York Botanical Garden's Herbarium (NY); Herbario del Instituto de Botánica Darwinion (SI); Herbario Privado Pedersen (PE); Missouri Botanical Garden's Herbarium at Saint Louis (actualmente la sigla es MO) (STL). Se citan según Holmgren et al. (1990), excepto el Herbario privado Pedersen, cuya sigla es PE.

El polen fue tratado para su observación con microscopio de luz (en adelante ML), mediante las técnicas de Wodehouse (1935) y de acetólisis de Erdtman (Nilsson \& Praglowski 1992), aunque mediante este procesamiento los granos se colapsan con facilidad. El residuo fue montado sobre portaobjetos con glicerina gelatina y sellado con parafina. Las medidas de la longitud y anchura de las díades (L), diámetro ecuatorial (E), y el espesor de la exina se basan en mediciones de 20 díades por ejemplar estudiado. Para su observación con microscopio electrónico de barrido (en adelante MEB) una parte del material de herbario fue deshidratado y secado en punto crítico; la otra parte fue montada sin tratamiento para la observación de cubierta polínica. Las muestras fueron luego cubiertas con oro paladio y examinadas con un microscopio JEOL JSM T-100. La terminología adoptada es la utilizada por $\mathrm{O}^{\prime} \mathrm{Neill}$ et al. (1997).

\section{RESULTADOS}

Los granos de polen son dispersados en díades. Se observaron tétrades decusadas antes de la antesis y abundante cubierta polínica que cubre los granos de polen, principalmente en la zona de contacto de las díades.

Observadas con ML, éstas miden de 21 a $31 \mu \mathrm{m}$ de longitud y de 12 a $18 \mu \mathrm{m}$ de ancho. Los granos individuales de las díades son 
radialmente simétricos, tricolpados, esferoidales a subprolados. La exina tiene un espesor de 0.7-1.4 $\mu \mathrm{m}$, es tectada, psilada, con una estructura infratectal formada por columelas simples bajo el tectum.

Observados con MEB, se puede ver que las díades se mantienen unidas por puentes de exina, (Figs. 1B, D, 2D), que representan una fuerte unión que resiste a la técnica de acetólisis (díades calimadas). La superficie de los granos de polen presenta ornamentación microequinada, a veces con mamelones en la base, rasgos que varían en ejemplares de una misma especie. Los granos son colpados, con colpos ornamentados desde 8 hasta $15 \mu \mathrm{m}$ de longitud. La ornamentación de la membrana del colpo consiste de elementos aislados de forma irregular cuyo tamaño es dos veces el de los procesos similares, de las zonas no aberturales. Las abeturas pueden estar alineadas entre los granos de la díade, o desalineadas.

En las cuatro especies estudiadas se registró la presencia de aberturas en forma de " $\mathrm{L}$ " $\mathrm{o}$ sea con una continuación transversal al colpo en uno de los granos de la díada. En algunos casos esta abertura puede continuarse como una espiroabertura (Fig. 2A).

\section{Descripciones de las especies}

Podostemum comatum Hicken. (Fig. 1AD- F): Díades de 21-30um de longitud por 14-17 $\mu \mathrm{m}$ de ancho; con paredes que muestran fusión parcial de las exinas no aberturales y abundante cubierta polínica. Granos con tendencia a una continuación transversal de la abertura , abertura en "L" (Fig. 1A) y aberturas a veces alineadas entre los granos de las díades. Colpos de $8-10 \mu \mathrm{m}$. Exina de $1 \mu \mathrm{m}$ de espesor, microequinada con procesos de diferentes tamaños. Elementos de la escultura sobre la pared no abertural en forma de microequinas cortas con mamelones; procesos de la pared abertural del doble de tamaño en relación con el resto, aislados o unidos de a pares y con mamelones.

Material estudiado: ARGENTINA. Misiones: Salto Iguazú, Rodríguez 791 (BA).
Salto Iguazú, Tur 2133 (LP). Salto Bosetti 10/ XI/ 95, Tur 7986 (LP). Salto Bosetti, Zavaro 360 (LP). Cataratas del Iguazú, Cabrera et al . 58 (CTES, LP). BRASIL. Paraná: río Iguassú, 20/II/1949, Schwartz 7553 (LIL).

Podostemum distichum Chodat et Vischer. (Figs. 1 B-C-E y G): Díades de 23-31 $\mu \mathrm{m}$ de longitud y de $12-18 \mu \mathrm{m}$ de ancho. Pared compartida de la díada con puentes de exina en las zonas no aberturales (Fig. 1B). Abundante cemento polínico, principalmente entre los granos de la díada (Fig. 1C). Colpos de $8-11 \mu \mathrm{m}$. Aberturas generalmente alineadas entre los granos de la díada, con ocasionales no alineadas (Fig. 1B, C). Exina de $1 \mu \mathrm{m}$ de espesor, microequinada con procesos de diferente tamaño. Elementos esculturales de la pared no abertural cortos y sin mamelones basales (Fig. 1E). Microequinas de la zona abertural dos veces más grandes que las del esto del grano, con mamelones en la base que, generalmente se unen de a pares por sus bases (Fig. 1G).

Material estudiado: ARGENTINA. Misiones: Iguazú, Parque Nacional Iguazú, November 1948, Meyer 17036 (LIL). Salto Bozzetti , 20/II/1970, Tur 1316 (LP, SI, STL en alcohol). Parque Nacional Iguazú, Cataratas, Salto Bozatti 20/II/70 Tur 7986 (LP); 21/ II/1992; Tur 1316 (LP), Tur et Guaglianone 2027 (LP, SI). Iguazú 10/9/1915, Osten et Rojas 8165, 8166 (BAF) Libertador General San Martín 16/II/1992 Tur et Guaglianone 1953 (LP, SI).San Javier, Tur 2122 (LP), Tur 1930 (LP), Tur B 2141 (LP), Tur 1930 (LP) \& Guaglianoe \& al. 946 (LP). Corrientes: Monte Caseros, 12/I/1953, Nicora 6318 (SI).

BRASIL. Paraná: Cataratas del Iguazú, 28/ IX/1967, Tur 1020, 1022 (STL). Cataratas del Iguazú, 27/IX/1967, Boelcke 13429 (BAA).

Podostemum muelleri Warm. (Fig. 2A, B, C, D): Díades de 22-30 $\mu \mathrm{m}$ de longitud y de $12-17 \mu \mathrm{m}$ de ancho. Pared de la díada compartida con fusión parcial de las exinas. Abundante cemento polínico entre los granos. Aberturas comúnmente alineadas entre los granos de las 


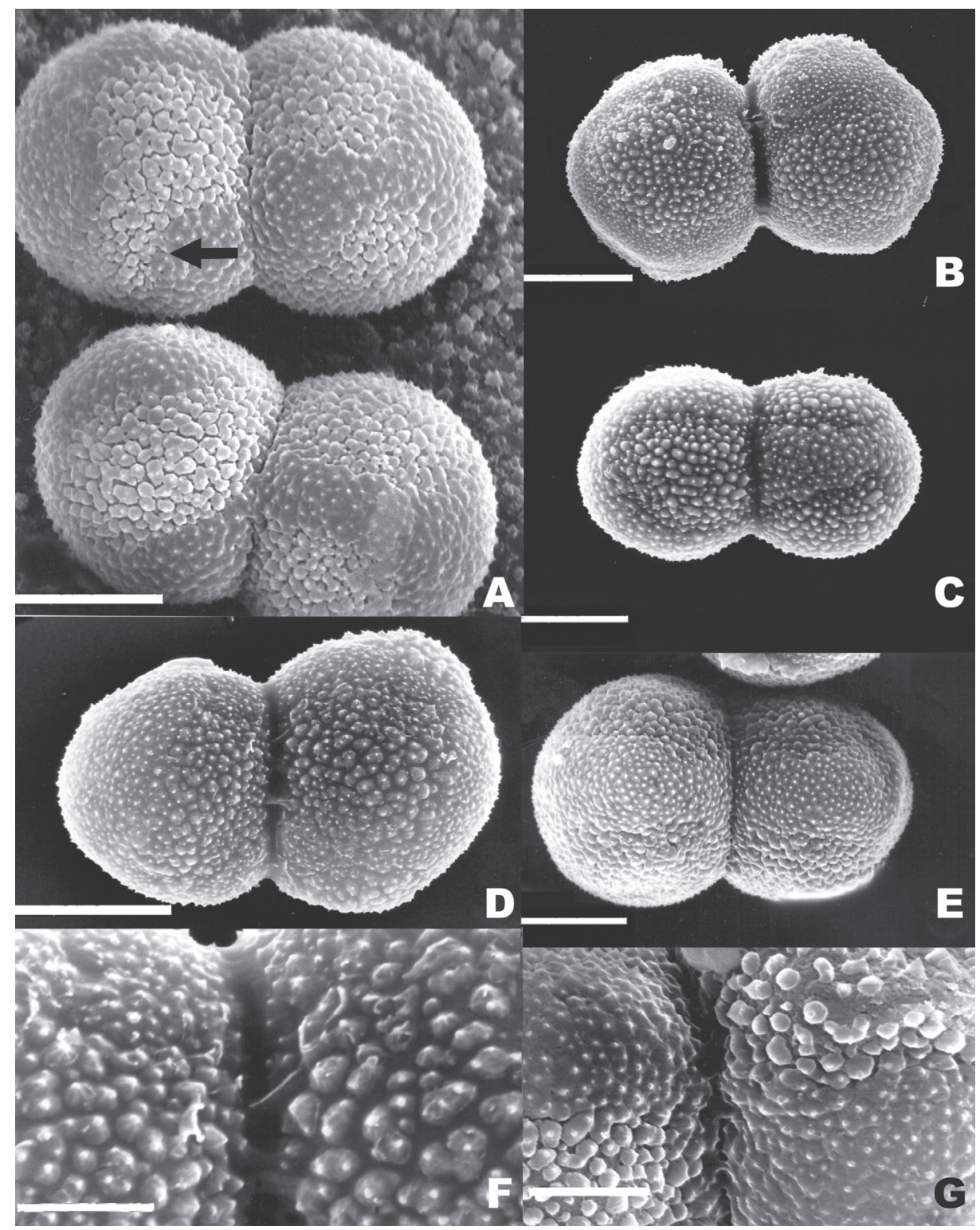

Fig. 1. Polen en especies de Podostemum: P comatum y P. distichum A- D- F- P. comatum. A: Colpo con abertura en "L" (flecha) procesos de las zonas aberturales dos veces más grandes que los no aberturales y con mamelones. Díades con colpos no alineados. D díada con colpos alineados, uno de los granos con abertura con tendencia a la "L", F. detalle de la ornamentación en la membrana del colpo. B- C-E-G. P. distichum B: díades con colpos no alineadas; pared compartida de la díada con puentes de esporopolenina en la zona no abertural. C: Granos con colpos alineados.E: díades con colpos alineados.G: detalle de membranas de los colpos con ornamentación mayor a la del resto del grano. Barra=A-D-F-G: $10 \mu \mathrm{m}$ y B-C: $8 \mu \mathrm{m}$.

Fig. 1. Pollen in species of Podostemum: P comatum and P. distichum A- D- F- P. comatum. A: Colpus with "L" aperture (arrow) colpus membrane with larger processes Dyads with no aligned colpus. D díada with aligned colpus, one of the pollen grains with "L" apertura F. detail of colpus membrane ornamentation B-C-E-G. P. distichum B: dyads with aperturas no aligned; the shared dyad wall shows bridges that partially fusion the exines. C: grains with aligned colps. E: grains with aligned colpus G: detail of colpus membrana ornamentation bigger than the rest of the pollen. Scale bar= A-D-F-G: $10 \mu \mathrm{m}$ and B-C: $8 \mu \mathrm{m}$. 


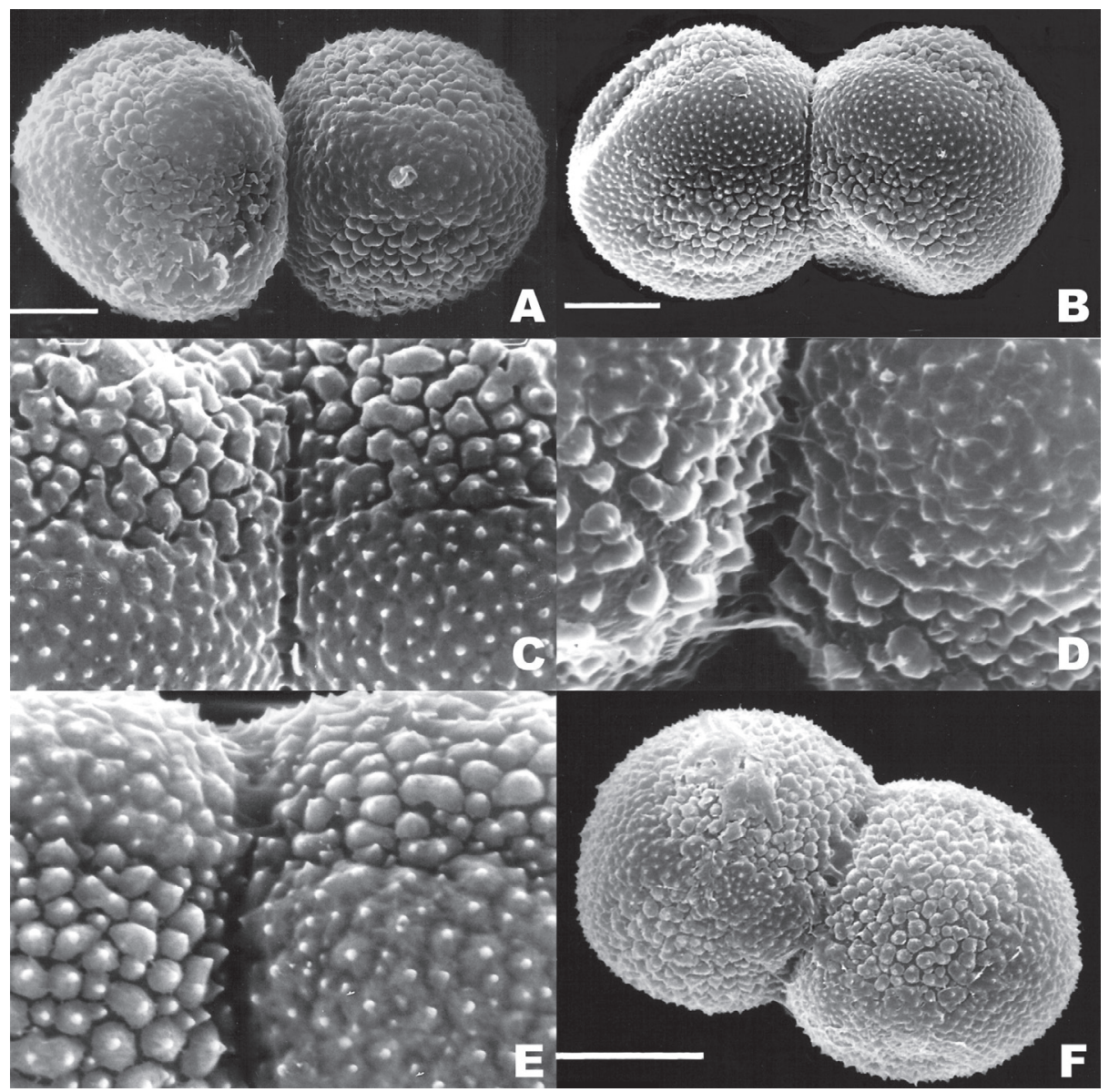

Fig. 2. Polen en especies de Podostemum:P. muelleri y P. rutifolium. A-B- C- D P. muelleri A- abertura en "L" en uno sólo de los granos de la díade; B granos de polen con colpos semi-alineados, $\mathrm{C}$ : microequinas aberturales similares a las del resto del grano pero de mayor tamaño y con mamelones D: pared compartida de las díades con fusión parcial de las exinas en zonas aberturales y no aberturales.. E: P. rutifolium detalle de aberturas no alineadas, ornamentación equinada de zonurales con mamelones F: Díada con aberturas no alineadas. Barra=A-B: $4 \mu \mathrm{m}$; F: $8 \mu \mathrm{m}$; C-D-E: la escala de F: $15 \mu \mathrm{m}$.

Fig. 2. Pollen in species of Podostemum: P. muelleri and P. rutifolium. A-B- C- D P. muelleri A- apertura in "L" in only one of the pollen grains of the dyads B pollen grains with semi-aligned colpus, C: microechinate sculpture in the apertural zone, similar to the rest of the grain but double size and with mamelonns D: shared dyad wall fusioned in apertural and non apertural zones. E: P. rutifolium detail of colpus membrana, equinate ornamentation of aperturales zones with mamelons $\mathrm{F}$ : Dyads with no aligned apertures. Scale bar: A-B: $4 \mu \mathrm{m}$; F: $8 \mu \mathrm{m}$; C-D-E: la escala de F: $15 \mu \mathrm{m}$.

díades. Granos de polen con tendencia a tener una abertura en "L" (Fig. 1A). Exina de $1 \mu \mathrm{m}$ de espesor, microequinada con procesos de diferente tamaño (Fig. 1H). Colpo de 8-10 $\mu$ m. Elementos esculturales de la pared no abertural cortos y sin mamelones basales. Elementos esculturales de la zona abertural microequinados, dos veces más grandes que los del resto del grano, con mamelones en la base y unidos de a pares.

Material estudiado: ARGENTINA. Misiones: Cainguás, 18/X/1975, Zuloaga et Deginani 561 (LP). Aristóbulo del Valle 15/ II/92, Tur et Guglianone 1948 (LP, SI). El Dorado , 13/II/70, Tur 1309 (LP, SI, STL), 13 / 
II/70, Tur 1313 (LP, SI, STL) \& Tur 2141 (LP).

Corrientes: Sto. Tomé Pedersen 9236 (C, LP, NY) \& 2147 (LP). Entre Ríos: Concordia, 10/ II/1967, Tur 954 (LP, SI, STL).

Podostemum rutifolium Warm. ( Fig. 2E, F):Díades de 23-28um de longitud por $12-17 \mu \mathrm{m}$ de ancho. Pared compartida de la díada con fusión parcial de la exina tanto en la zona abertural como en el resto del grano. Colpos de $9 \mu \mathrm{m}$. Aberturas normalmente alineadas entre los granos de las díades o desalineadas (Fig. 2F). Exina de $1 \mu \mathrm{m}$ de grosor, microequinada con procesos de diferente tamaño (Fig. 2E). Los elementos esculturales sobre la pared no abertural cortos y con mamelones basales. Microequinas de la zona abertural similares en forma pero dos veces el tamaño de las del resto del grano.

Material estudiado: ARGENTINA. Misiones: Apóstoles, Tur et Guaglianone 967 (LP). Iguazú, 26/I/1983, Guaglianone et al. 997 (LP. SI). Iguazú, Guaglianone et al. 999 (LP, SI).Iguazú, 20/II/1970, Tur 1307 (LP, SI). Parque Nacional Iguazú 21/II/1992 Tur \& Guaglianone 2025 (LP, SI). Salto dos Hermanas, Tur 1137 (LP) ,1022 (STL) 1316 (LP) \& Tur 1137 (LP) . BRASIL. Paraná: Río Iguassú 10/ II/1949, Schwartz 7555 (LIL).

\section{DISCUSIÓN}

Se presentan las primeras descripciones palinológicas de cuatro especies neotropicales de Podostemum: P. comatum, P. distichum, $P$. muelleri y $P$. rutifolium. Las semejanzas halladas en los rasgos morfológicos presentes en el polen de las especies estudiadas coincide con la solidez filogenética asignada al grupo (Tur 1997).

La morfología de los granos individuales de las díades son similares a los descritos para otros géneros de la familia, como Apinagia Tul. y Mourera Aubl. (Passarelli et al. 2002a, 2002b) y lo observado en Marathrum Bonpl. (Passarelli, datos no publicados). La ausencia de mamelones en las zonas interaberturales observada en $P$. ceratophyllum Michx por O'Neill et al. (1997) no coincide con lo observado aquí, pero esos rasgos parecen tener escaso valor diagnóstico ya que difieren en especímenes de la misma especie, algo observado también en Apinagia (Passarelli et al. 2002a).

El carácter palinológico más interesante es la presencia de aberturas que se continuan transversalmente, llamadas aquí en forma de "L" y que podría tratarse de un paso previo a la espiroabertura. La presencia de espiroaberturas ha sido citada para esta familia por primera vez por Osborn et al. (2000) para Marathrum schiedeanum (Cham.) Tul., quienes las consideraron un carácter avanzado.El género Podostemum crece en rápidos o en cataratas, sobre rocas, por lo tanto su morfología responde a varios mecanismos de adaptación. Furness (1985) señala que la espiroabertura incrementa las posibilidades de sitios de germinación, este concepto es también aplicable a la abertura en "L" observada en Podostemum, algo que es importante por ser éste un género de floración y antesis muy breves; en general un día de antesis durante la estación seca (observaciones fenológicas de una de las autoras) lo que lleva a la necesidad concomitante de una germinación rápida. Furness \& Rudall (2004) consideraron que un incremento en el número de aberturas de los granos de polen es una potencial ventaja selectiva, que habría en las angiospermas una tendencia a un incremento en el número de aberturas polínicas y que el cambio de posición de polar a ecuatorial en las eucotiledóneas pudo conducir al éxito en la radiación y rápida adaptación de este grupo, especialmente en que se encuentran bajo una presión de selección, algo que podría incluir a Podostemum. Además, la presencia de espiroaberturas o aberturas similares permitiría la resistencia a un ambiente con variaciones hídricas extremas teniendo función harmomégata.

La dispersión en díades es inusual en angiospermas y representa también una ventaja ya que al menos dos granos estarán en contacto con el estigma. La observación de tétrades decusadas permite explicar el origen 
de esta unidad de dispersión en Podostemum. La subdivisión en subfamilias, Podostemoideae y Tristichoideae, la primera con tétradas producidas en forma sucesiva y la segunda en forma simultánea (Jäger-Zürn et al. 2005) indican con pocas dudas que este carácter es variable, ya que Podostemoideae presenta ambos tipos de microsporogénesis. Furness \& Rudall (2004) sugirió que las eucotiledóneas parecen tender a la formación de tétrades tetraédricas simultáneas, por lo que la presencia de tétrades decusadas (sucesivas) en el género Podostemum lo distancia del resto del grupo.

La presencia de abundante cubierta polínica estaría relacionada con el ambiente y con la polinización no zoofila, ya que las flores verdosas, sin perfume, carecen de atractivos para este tipo de polinización. Philbrick et al. (2006) registraron autogamia en preantesis, en P. ceratophyllum y P. weddellianum (Tul.) C.T. Philbrick \& Novelo. Como las autoras observaron que las flores presentan antesis en el momento de sequía, es posible que la cubierta polínica participe tanto en el reconocimiento específico como en la protección a la desecación del grano de polen.

\section{AGRADECIMIENTOS}

Las autoras agradecen a Cristina H. Rolleri los valiosos comentarios sobre este trabajo y la lectura crítica del manuscrito. Se reconocen además los comentarios aportados por los revisores que permitieron mejorar el manuscrito. Este estudio fue realizado con el aporte del Consejo Nacional de Investigaciones Científicas y Técnicas (CONICET, PIP 5044-LP) institución a la que pertenece la segunda autora y el Programa de Incentivos para Docentes Investigadores de la Universidad Nacional de La Plata, al que se adscribe la primera. Se agradece además, la colaboración técnica de Patricia Sarmiento del Servicio de Miroscopía Electrónica de Barrido del Museo de Ciencias Naturales, Universidad Nacional de La Plata.

\section{RESUMEN}

La morfología del polen de cuatro especies neotropicales del género Podostemum (Podostemaceae) se describe aquí por primera vez mediante microscopía de luz y electrónica de barrido, utilizando material deshidratado en punto crítico. Se analizaron ejemplares de $P$. comatum, $P$. distichum, P. muelleri y P. rutifolium. Los granos de polen son dispersados en díades. Se encuentra abundante cobertura polínica, principalmente en la zona de contacto entre los dos granos de la díada. En la pared compartida entre los granos se presentan puentes de ectexina que fusionan parcialmente las exinas. Los granos individuales son radialmente simétricos y tricolpados. En algunas díades las aberturas están alineadas entre los granos y en otras no. Se describe por primera vez la condición abertura transversal, que fue observada en las cuatro especies estudiadas, y que se interpreta como tendencia del género a espiroabertura. La escultura de la exina es microequinada con mamelones en la base. Los procesos ornamentales son de diferente tamaño. La membrana del colpo tiene una ornamentación similar a la pared adyacente del grano, pero los procesos tienen mayor tamaño. Se observaron tétrades decusadas en las etapas previas a la antesis, un dato hasta el momento no registrado para el género.

Palabras clave: morfología polínica, PodostemumPodostemaceae, díades, abertura tricolpada, espiroabertura, plantas acuáticas, flora neotropical

\section{REFERENCIAS}

Ancibor, E. 1990. Anatomía de las especies argentinas de Podostemum Michx.(Podostemaceae). Parodiana 6: 31-47.

Cook, C.D.K. 1996. Aquatic plant book. SPB, Amsterdam, Holanda.

Furness, C.A. 1985. A review of spiroaperturate pollen. Pollen et Spores 27: 307-320.

Furness, C.A. \& P. J. Rudall. 2004. Pollen Aperture Evolution-a crucial factor for eudicot success?. Trends Plant Sci. 9: 154-158.

Holmgren, P.K., N.H. Holmgren \& L. C.Barnett. 1990. Index Herbariorum. Part. I: The Herbaria of the World, Jardín Botánico de Nueva York, Nueva York, EEUU.

Jäger-Zürn, I., A. Novelo \& T. Philbrick. 2005. Microspore development in Podostemaceae-Podostemoideae, with implications on the characterization of the subfamilias. Plant Syst. Evol. 256: 209- 216. 
Kita, Y.\& M. Kato. 2001. Infrafamilial phylogeny of the acquatic angiosperm Podostemaceae inferred from the nucleotide sequence of the matk gene. Plant Biol. 3: $156-163$.

Les, D., T. Philbrick \& A. Novelo. 1997. The phylogenetic position of river-weeds (Podostemaceae): Insights from rbcL sequence data. Aquatic Bot. 57: 5-27.

Nilsson, S. \& J. Praglowski. 1992. Erdtman's handbook of palynology.Munksgaard. Copenhagen, Dinamarca.

O’Neill, S., J. Osborn, C.T. Philbrick \& A. Novelo. 1997. Comparative pollen morphology of five New World genera of Podostemaceae. Aquatic Bot. 57: 133-150.

Osborn, J., S. O’Neill \& G. El-Ghazaly. 2000. Pollen morphology and ultrastructure of Marathrum schiedeanum (Podostemaceae). Grana 39: 221-225.

Passarelli, L.M, S.Girarde \& N. M. Tur. 2002a. Palynology of South American Podostemaceae. I. Apinagia Tul. Grana 41: 10-15.
Passarelli, L., S. Girarde \& N.M. Tur. 2002 b. Caracteres Palinológicos de las especies de Mourera y Tristicha (Podostemaceae) que crecen en Argentina. Bol. Soc. Arg. Bot. 34: 231-234.

Philbrick, C.T.\& A. Novelo. 1995. New World Podostemaceae: Ecological and evolutionary enigmas. Brittonia 47: $210-222$.

Philbrick, T. \& A. Novelo. 2004. Monograph of Podostemum. Syst. Bot. Monogr.70: 1-106.

Philbrick, T., M.Vomela \& A. Novelo. 2006. Preanthesis cleistogamy in the genus Podostemum (Podostemaceae). Rhodora 108:195-202.

Tur, N.M..1997. Taxonomy of Podostemaceae in Argentina. Aquatic Bot. 57: 213-241.

Wodehouse, R.P. 1935. Pollen grains. Mc Graw-Hill, Nueva York, Nueva York, EEUU. 